\title{
LINGUISTIQUE DU TEXTE ET DE L'ÉCRIT - STYLISTIQUE
}

\author{
Georges Molinié \\ Paris-IV (Paris-Sorbonne)
}

\begin{abstract}
On peut prendre la désignation de cette thématique de plusieurs points de vue, ce dont ne se sont pas fait faute les auteurs de communication. On peut par exemple réfléchir sur ce que c'est que la linguistique du texte, dans le sens des travaux de Jean-Michel Adam ou de Dominique Maingueneau, ou sur ce que c'est que la linguistique de l'écrit, dans la lignée de ceux de Joëlle Gardes-Tamine ou d'Anne HerschbergPierrot, ou sur ce que c'est que la sémiotique du texte dans le sillage des questionnements de Sémir Badir, ou sur ce que c'est que la stylistique, avec les propositions du genre de celles d'Anna Jaubert, ou de Madeleine Frédéric, ou de Gilles Philippe, ou d'Éric Bordas, ou des miennes, pour ne citer que quelques noms emblématiques parmi d'autres également très considérables. Vaste panorama, comme vaste programme; diversité des approches, et, tout ensemble, quelque chose qui semble aimanter communément. Et encore, n'a-t-on point évoqué le double continent, récemment revisité et re-balisé, de l'analyse du discours et de la socio-critique, aux marges ou à l'horizon du nôtre en ce lieu, susceptible d'en former l'idéologie englobante, tout en acceptant en son propre sein un partage à dominante justement méthodologico-idéologique. Ce qui revient à admettre comme décisifs les partis pris de méthode et des choix objectaux.
\end{abstract}

Je préfère, à titre de crayon pour cette étape provisoire, réfléchir sur les conditions et sur la portée heuristiques, je n'ose, malgré mes propres thèses aujourd'hui, dire herméneutiques, du rassemblement de ce titre.

Qu'il y ait un rapport entre linguistique et stylistique, c'est pour moi un acquis ancien, mais non absolument ni nécessairement originel, dans la mesure où, avant Bally et parallèlement à son œuvre, se sont développées des stylistiques des arts non-verbaux, qui ne sont pas sans prégnance sur l'idée stylistique, ne serait-ce que par deux déterminations fondamentales: le programme d'un faisceau de caractères formels, de traits expressifs, qui à la fois conduit à la définition d'un style, et induit une pensée du style, comme caractéristique ; et l'affinité atavique entre stylistique et esthétique des arts.

On peut (on doit ?) poser ici que se déploie un double mouvement.

La stylistique (sans doute faut-il aussi penser qu'il ne s'agit pas davantage, en l'occurrence, $d u$ stylistique), comme praxis et comme méthode, s'appuie sur la linguistique, en exploitant la totalité de ses domaines, de ses outils, de ses cheminements conceptuels, voire de ses problématiques. Mais il est clair que l'on doit chercher alors quelle est l'orientation heuristique d'une telle convocation et d'un tel programme - sauf à étudier l'emploi du subjonctif chez Baudelaire comme exemple ou comme témoin de l'emploi du subjonctif dans le français du XIX ${ }^{\mathrm{e}}$ siècle ; mais le subjonctif dans la poésie française du $\mathrm{XIX}^{\mathrm{e}}$ siècle, mais le subjonctif comme marqueur de l'écriture de Baudelaire? On peut poser les mêmes questions à propos du participe présent chez Claude Simon. L'orientation vise apparemment une particularité, collective ou individuelle, générique ou singulière. Et elle peut s'accrocher à la détermination d'une particularité liée à la réception d'une certaine artistisation - à condition de ne pas oublier qu'il n'existe pas, à mon avis, d'essence d'une quelconque pragmatique artistique, mais que n'importe quelle praxis langagière, selon des conditions spécifiques de réception, est susceptible d'un ressentiment, privé ou collectif, d'artistisation. Cette particularité visée par la démarche stylistique revient forcément à jouer sur l'articulation structure / réalisation, langue / usage, ce qu'il serait vraisemblablement plus rentable d'appréhender en terme hjelmslévien de manifestation. La stylistique s'intéresse aux manifestations d'un langage, et donc à ses effets de valeurs.

En sens inverse, avec la linguistique, du texte et de l'écrit, comme base de l'analyse stylistique, on a la forte spécification d'une stylistique du verbal (par opposition aux stylistiques d'autres sémioses, c'est-à- 
dire d'autres langages). Mais la spécification ici présentée est encore plus restrictive : on n'a pas dit largement $d u$ discours, même si l'on implicite, j'imagine, qu'il s'agit forcément de discours sociaux : on a exclu l'oral, en posant, comme une sorte d'hendiadyn, du texte et de l'écrit, au sens, que je décide de comprendre, qu'il existe une affinité structurale entre textualité et scipturarité, ce qui induit d'ailleurs en outre l'affinité avec un troisième terme, la littérarité (même si l'on se rappelle les commentaires précédents sur la gradualité et sur l'instabilité des réceptions d'artistisation; même si l'on reconnaît la vitalité sémiotique du concept de littérature orale: il est clair que le littérarisable a vocation au scripturaire). On a ainsi comme objet dur, pour nous, de la langue française, avec ses divers niveaux de composantes construisibles et descriptibles à l'analyse, ce qui relève précisément de la linguistique française. Mais avec la spécification que l'on considère exclusivement la forme discours textuel (ce qui implique de l'écrit).

On pourrait alors se demander si cette double détermination, ou cette détermination doublement prédiquée, ne constitue pas, de soi, une classe stylistique, du moins un horizon stylistique; si cette classe ou cet horizon n'épuiseraient pas la caractéristique stylistique (de l'objet); si la linguistique « du texte et de l'écrit », comme du scripturaire et comme du textuel, n'est pas une stylistique. Ce sont en tout cas des formes de manifestation.

L'une d'elle, la scripturaire, obéit évidemment à un nombre d'une certaine façon fini (en tout cas finissable, fût-ce par emboîtement d'ensembles homogènes) de traits distinctifs. C'est au point que l'on pourrait se demander si une grammaire de l'écrit, indépendamment de la théorie que sa systématique induit, ne fonctionne pas aussi, d'un autre point de vue, comme une des stylistiques possibles d'une langue (en l'occurrence, le français d'aujourd'hui). C'est une autre façon de poser la question de la marque et de la valeur différentielle, par rapport à la pensée d'une langue.

La chose est semble-t-il plus compliquée avec la manifestation textuelle. Je serais forcément tenté de la prendre comme une forme stylistique de base, une sorte de $u r$-manifestation, surtout si j'en relie la conception à mon idée de macro-texte, qui est une manière de pointer le caractère terriblement plastique et totalement construit de cette prétendue objectalité.

Ce qui est en cause à ce point, c'est, de fait, la question, qui est un vrai problème méta-sémiotique (à tout le moins méta-linguistique), de la consubstantialité de l'ordre des déterminations. Avec la textualité, on a évidemment un constituant sémio-linguistique majeur du stylistique ${ }^{\mathrm{i}}$ : nous sommes, je crois, largement d'accord pour affirmer, conformément aussi aux positions de François Rastier, que le texte constitue l'un des milieux phénoménologiques dominants à la formation, à la détection et à l'évaluation de quelque structuration stylématique que ce soit. C'est le texte qui, à l'écrit, définit essentiellement le bassin d'attraction où peut se produire le jeu de saillance de valeurs capable de générer, localement, graduellement, variablement, mais à chaque réception décisivement, la sensation du stylistique, la réaction de stylicité — pour quelqu'un, pour quelques-uns.

Mais on a aussi de bonnes raisons, également bien connues, de soutenir que la textualité n'est pas une qualité triviale, que le texte n'est pas un donné, qu'il n'y a nulle évidence à cette objectalité supposée. Bien sûr, doit-on raisonner en terme de cohésion et/ou de cohérence, en terme d'unité, en terme de limite ; la pensée du texte, on l'a dit, a à voir avec la pensée des genres, avec celle de la phrase, ou sans doute plus puissamment, avec celle de la période. Mais on sait bien qu'on ne parle pas forcément exactement de la même chose quand on parle d'un texte de Boileau, d'un texte de Claude Simon, d'un texte sur un mur, ou d'un texte de François Mitterrand. Tout texte, c'est-à-dire toute empirie scripto-discursive désignée comme texte, est le résultat au mieux (au plus épistémologique) d'une décision heuristique, au plus communément d'une rencontre (ce qui n'est pas moins important). On peut passer le long d'interminables suites discursives sans jamais s'y fixer (c'est même la situation générale) : la fixation dépend de l'attention sensori-perceptive d'une situtation singulière de réception. Certaines situations sont plus largement socialement codées, d'autres plus individuelles et plus instables. On ne peut même pas s'en tenir aux limites matérielles fixées à la production, puisqu'on est toujours libre de les transgresser, dans un sens ou dans un autre, à réception; pas davantage ne saurait suffire, à la réalisation d'une pertinence textuelle immanente, l'architecture de déterminations sémio-linguistiques internes, puisque celles-ci 
dépendent d'opérations cognitives qui en conditionnent la construction dans la focalisation de la rencontre, dans le phéno-social de telle ou telle empirie. Tout au plus pourrait-on concevoir davantage d'autonomie, même relative, aux déterminations génériques, ou rhétorico-pragmatiques, plus cosubstantielles à la matérialité de tel ou tel discours que son appréhension en texte, comme texte.

D'une certaine façon, la textualité est à la fois constituante du stylistique et constituée par l'approche stylistique.

En prenant un peu de hauteur, relativement à l'objet même du colloque et sans se restreindre à la linguistique française en tant que linguistique du français, on aurait envie de se demander s'il ne serait pas opportun de rouvrir une dialectique fondamentale, ou d'ouvrir une nouvelle dialectique fondamentale, de manière à poser le stylistique comme herméneutique du langagier, en présentant alors l'idée de texte comme homologue à l'idée saussurienne de langue, dans la perspective de l'appréhension des systèmes de valeurs.

\footnotetext{
${ }^{\mathrm{i}}$ Je change maintenant de niveau de réflexion, n'absorbant plus frontalement, comme je viens de proposer de le faire, les considérants de linguistique textuelle, comme textuelle, dans la problématique stylistique. Mais on va voir qu'on y revient inévitablement.
} 